\title{
Does nonadherence to local recommendations for empirical antibiotic therapy on admission to the intensive care unit have an impact on in-hospital mortality?
}

\author{
This article was published in the following Dove Press journal: \\ Therapeutics and Clinical Risk Management \\ I July 2009 \\ Number of times this article has been viewed
}

\author{
Jean-Luc Baudel' \\ Jacques Tankovic ${ }^{2}$ \\ Fabrice Carrat ${ }^{3}$ \\ Cécile Vigneau' \\ Eric Maury ${ }^{1,3}$ \\ Valérie Lalande ${ }^{2}$ \\ Bertrand Guidet ${ }^{1,3}$ \\ Georges Offenstadt ${ }^{1,3}$
}

'Service de Réanimation Médicale; ${ }^{2}$ Service de Bactériologie-Virologie, Universite Pierre et Marie Curie, Paris, France; ${ }^{3}$ Hôpital Saint-Antoine, Assistance Publique-Hôpitaux de Paris, Paris, France
Correspondence: Jean-Luc Baudel Service de Réanimation Médicale, Hôpital Saint-Antoine, 184 rue du Faubourg Saint-Antoine, 75 57I Paris Cedex 12, France

Tel +33 I49 2830 I3

Fax +33 $149282 \quad 145$

Email jean-luc.baudel@sat.aphp.fr
Objective: 1 / To evaluate if empirical antibiotic prescription on admission to our intensive care unit (ICU) respects the local recommendations for antibiotic prescription and to identify predictors of nonadherence to these guidelines. 2 / To assess whether nonadherence to the guidelines is associated with increased in-hospital mortality due to the initial infection.

Materials and methods: This was a prospective six-month observational study performed in a 14-bed medical ICU. Patients were included if they received curative antibiotic therapy on admission. Respect of the local treatment recommendations was evaluated according to adherence to the local empirical guidelines defined in a 80-page booklet which is given in our hospital to every physician.

Results: Among 132 antibiotic prescriptions, 21 (16\%) were unjustified (absence of infection), $17(13 \%)$ were microbiologically documented at admission, and nine $(7 \%)$ were given for infections from unknown origin. Among the 85 (64\%) empirical prescriptions that could be evaluated for adherence to local recommendations, nine $(11 \%)$ were inappropriate and $76(89 \%)$ appropriate. In univariate analysis hospital-acquired infection was the sole predictor of inappropriate treatment $(\mathrm{p}=0.0475)$. Independent predictors of in-hospital mortality due to the initial infection were inappropriate empirical treatment (odds ratio $[\mathrm{OR}]=14.64,95 \%$ confidence interval [CI]: 2.17-98.97; $p=0.006)$, prescription of fluoroquinolones $(\mathrm{OR}=8.22$, 95\% CI: $1.88-35.95 ; \mathrm{p}=0.005$ ) and a higher Simplified Acute Physiology Score II score (per one-point increment $(\mathrm{OR}=1.04,95 \% \mathrm{CI}: 1.01-1.07 ; \mathrm{p}=0.02)$.

Conclusion: Nonadherence to local empirical antibiotic therapy guidelines was associated with increased in-hospital mortality due to the initial infection.

Keywords: antimicrobial therapy, appropriateness, mortality, intensive care unit

\section{Introduction}

It is widely accepted that prompt, appropriate antimicrobial chemotherapy is an important determinant of outcome in infected critically ill patients. The impact of treatment appropriateness on survival has been well documented during the course of ventilatorassociated pneumonia, ${ }^{1-4}$ bloodstream infections, ${ }^{5-7}$ sepsis, severe sepsis, and septic shock. ${ }^{8-11}$ Although the impact of treatment appropriateness has not been studied specifically in patients infected on admission to the intensive care unit (ICU), one might imagine that appropriate antimicrobial therapy is particularly warranted in this situation.

In all the above-mentioned studies, ${ }^{1-11}$ appropriateness of antibiotic therapy was only judged according to microbiological documentation. Undocumented infections 
thus could not be evaluated. This represents an important limitation of the above-referenced studies, because $30 \%$ of hospital-acquired infections and $45 \%$ of community-acquired infections remain undocumented in ICUs. ${ }^{12}$ As our local anti-infective committee has established recommendations for empirical antibiotic therapy including clinically suspected microbiologically undocumented infections, we used them as a method of evaluating the appropriateness of empirical antibiotic prescriptions given for patients on admission to the ICU. Using this definition of treatment appropriateness, we characterized the quality of antibiotic treatment on admission, the factor(s) associated with treatment inappropriateness, and the impact of inappropriateness on in-hospital mortality attributed to the initial infection.

\section{Materials and methods Setting and patients}

This prospective study was carried out during a six-month period (February 12 to July 25, 2003) in the 14-bed medical ICU of Saint-Antoine Teaching Hospital in Paris, France. All patients receiving empirical curative antibiotic therapy in the first 24 hours following admission to the ICU were eligible. Empirical therapy was started after the collection of a minimum of two peripheral blood samples in $100 \%$ of cases, and of other clinically indicated microbiological samples in $88 \%$ of cases (bronchoalveolar lavage fluid, pleural fluid, urine, cerebrospinal fluid, pus, etc.).

\section{Data collection}

The following data were systematically recorded: date and time of admission, age, gender, prior hospitalization in the previous year, origin of the patient, ongoing antimicrobial chemotherapy on admission and eventual modification of that treatment, Simplified Acute Physiology Score (SAPS II), ventilatory support, vasopressor administration, indication(s) for antimicrobial chemotherapy, community-acquired or hospital-acquired infection (classified as hospital-acquired if diagnosed more than 48 hours after hospital admission), antibiotics prescribed, time between admission and administration of antibiotic therapy, and initiation of antibiotic therapy during the day time (between $8 \mathrm{AM}$ and $8 \mathrm{PM}$ ) or at night. After 48 hours, we collected the microbiological data and recorded whether antimicrobial treatment was modified or not.

\section{Justification and appropriateness of therapy}

One week after ICU admission, all the files were reevaluated by two investigators (JLB and JT). This provided a classification of antibiotic treatments: $1 /$ justified (presence of infection) or not (no infection); 2 /prescribed for a correctly identified source of infection or not (site of infection not found or misdiagnosed site of infection); 3/appropriate or not (appropriateness being defined as respect of the published local recommendations for empirical antibiotic treatment. This could be done only for clinically, biologically and/or radiologically established but microbiologically undocumented infections). Appropriateness was evaluated by the investigators (JLB, JT) and concerned both the molecules employed and their dosages. Our published local guidelines consist of an 80-page booklet updated every two years, which covers almost all infection sites. For example, concerning community-acquired broncho-pulmonary infections, the different presentations are defined (acute bronchitis, acute exacerbation of chronic obstructive pulmonary disease, acute pneumonia of the young adult [not severe or severe], acute pneumonia after aged 75 years [not severe or severe or with comorbidities] aspiration pneumonia) and, for each one, a first-line treatment is proposed (molecules and dosages) as well as some alternatives (two to four depending on the infection). This booklet is given personally to each clinician of our hospital. The recommendations present in this booklet are based on the practice guidelines of the Infectious Diseases Society of America and of the Société de Pathologie Infectieuse de Langue Française. Treatment was considered inappropriate when it did not respect these recommendations in terms of molecules and dosages, and when this resulted in a treatment inactive against the pathogens most frequently responsible for the type of infection presented by the patient.

\section{Outcome}

We recorded in-hospital mortality related to the infection present on ICU admission. This assessment was done independently by the two main investigators; in the absence of consensus the chart was re-evaluated by the two investigators or by the entire medical staff of the ICU until a conclusion was drawn.

\section{Statistical analysis}

The chi-squared test or Fisher's exact test was used to compare qualitative variables, and the Mann-Whitney test was used for quantitative variables. Logistic regression models were used to identify risk factors for in-hospital mortality caused by the the initial infection. Characteristics with $p$ values below 0.20 in univariate analysis were included in multivariate models based on a backward elimination procedure. All statistical tests were two-sided, with a type I error of $5 \%$. 


\section{Results}

During the study period, 374 patients were admitted to the ICU. One hundred twenty seven of them (34\%) received curative antibiotic therapy within the first 24 hours. One hundred twenty two (96\%) of these patients were treated for one focus of infection, and five patients for two foci (total 132 prescriptions). The baseline characteristics of the patients are shown in Table 1. Patients who were given antibiotics on admission were older, had a higher SAPS II score, a longer ICU stay, and required a higher work load.

File review showed that $21(16 \%)$ of the 132 prescriptions were unjustified because of absence of infection. The characteristics of the 111 justified curative antibiotic prescriptions, which concerned 107 patients (four patients were treated for two infections) are shown in Table 2. Sixty-one (57\%) of these 107 patients were outpatients, 39 (43\%) were inpatients. 45 of these 107 patients (42\%) had already received antibiotics on admission. This antibiotic therapy was frequently modified on admission $(31 / 45,69 \%)$, in most cases $(81 \%)$ because it was considered inappropriate by the prescriber. Antibiotic therapy begun on admission was always reviewed 48 hours later and this led to treatment changes in $55 \%$ of the cases: narrowing (49\%), escalation (6\%). Most cases of treatment de-escalation were explained by the availability of microbiological data at 48 hours. Concerning treatment escalation, in most cases treatment was changed because on admission the nosocomial character of the infection had not been taken into account.

We first studied the appropriateness of the prescriptions according to our guidelines. Among the 111 justified prescriptions, $17(15 \%)$ were microbiologically documented at admission and nine ( $8 \%$ ) with either no identification of the source of infection (eight cases) or a misdiagnosed site of infection (one case: intra-abdominal infection misdiagnosed as pneumonia). For these 26 cases, our local empirical treatment guidelines were not useful for the prescriber and thus we did not include them in the evaluation.

We thus determined the adherence to the guidelines of 85 empirical antibiotic prescriptions for correctly diagnosed infections ( $77 \%$ of the 111 justified prescriptions): nine were classified as inappropriate (11\%) and 76 appropriate (89\%). All the nine inappropriate treatments were related to an inadequate choice of antimicrobial agent(s), but not to a wrong dosage. Univariate analysis (Table 3 ) showed that treatment of a hospital-acquired infection was the sole factor significantly associated with nonadherence to our local guidelines.

Thirty-nine of these 85 prescriptions (46\%) were secondarily documented following admission. Thus the total percentage of microbiologically documented infections at 48 hours was $50 \%$ (56/111, 17 on admission plus 39 secondarily).

Our last and main objective was to appreciate whether nonadherence to our local treatment guidelines was associated with in-hospital mortality due to the initial infection. Among the 26 in-hospital deaths ( $23 \%$ of the study population), the initial infection was considered to be the cause of death in 13 cases $(50 \%)$. Four factors were significantly associated with mortality due to the initial infection: 1 /usage of intravenous epinephrine or norepinephrine; $2 /$ empirical treatment inappropriate or prescribed for an infection of unknown origin; 3/usage of certain classes of antibiotics (fluoroquinolones and hospital-used beta-lactams); 4/high SAPS II score (Table 4). Mortality was much higher in case of inappropriate empirical treatment $(44 \%, 4 / 9)$ and of empirical treatment without localization of the infection source $(33 \%, 3 / 9)$ than in case of appropriate empirical treatment $(5 \%, 4 / 73)$ and microbiologically documented treatment $(12 \%, 2 / 16)$. There was a trend towards increased in-hospital mortality in case of modification of the antimicrobial chemotherapy given prior to admission, but this did not reach statistical significance $(\mathrm{p}=0.13)($ Table 4$)$.

Table I Baseline characteristics of the patients admitted to the ICU during the study period classified in two groups: study patients who were given curative antibiotic therapy on admission and all other patients

\begin{tabular}{|c|c|c|c|}
\hline Variable $^{a}$ & Study patients $(n=127)$ & Other patients $(n=247)$ & $\mathbf{p}$ \\
\hline Age (years) & $60.8 \pm 16.7$ & $55.7 \pm 19.0$ & $<0.01$ \\
\hline Male to female ratio & 1.50 & 1.44 & ns \\
\hline SAPS II & $41.9 \pm 19.3$ & $36.9 \pm 22.4$ & $<0.01$ \\
\hline Length of ICU stay (days) & $8.6 \pm 13.3$ & $3.0 \pm 4.3$ & $<0.01$ \\
\hline Work load: Omega score ${ }^{b}$ & $|26.8 \pm 18|$ & $49.5 \pm 71.2$ & $<0.01$ \\
\hline ICU mortality & $12.5 \%$ & $11 \%$ & ns \\
\hline
\end{tabular}

Notes: ${ }^{a}$ Values are expressed as mean \pm SD, except for male to female ratio and ICU mortality; ${ }^{\circ}$ The Omega Score is composed of therapeutic items accorded I to 10 points, and is divided into three categories as follows: Category I, items entered only at the time of their first application; Category 2, items entered at each application; and Category 3 , items entered every day of application. The total score, which covers the entire length of stay, is calculated by adding the points obtained in the three categories. Abbreviation: SAPS II, simplified acute physiology score. 
Table 2 Description of the justified curative antibiotic prescriptions administered on admission to the ICU $(\mathrm{n}=\mathrm{II} \mathrm{I})$

\begin{tabular}{|c|c|c|}
\hline Aspect & \multicolumn{2}{|l|}{ Data } \\
\hline Number of treatment indications per patient & \multicolumn{2}{|c|}{ One for 103 patients, two for 4 patients: III prescriptions } \\
\hline Number of prescriptions for community-acquired infections & \multicolumn{2}{|l|}{$84(76 \%)$} \\
\hline Number of prescriptions for nosocomial infections & \multicolumn{2}{|l|}{$27(24 \%)$} \\
\hline Empirical treatment for correctly diagnosed infection & \multicolumn{2}{|l|}{$85(77 \%)$} \\
\hline Empirical treatment for undiagnosed or misdiagnosed infection & \multicolumn{2}{|l|}{$9(8 \%)$} \\
\hline Microbiologically documented treatment & \multicolumn{2}{|l|}{17 (I5\%) } \\
\hline \multirow[t]{3}{*}{ Number of monotherapies, bitherapies, and tritherapies } & Monotherapies: & $4 \mathrm{I}(37 \%)$ \\
\hline & Bitherapies: & $65(58.5 \%)$ \\
\hline & Tritherapies: & $5(4.5 \%)$ \\
\hline \multirow[t]{22}{*}{ Classes of antibiotics prescribed, $n=180$} & Beta-lactams: & $102(57 \%)$ \\
\hline & Co-amoxiclav: & 67 \\
\hline & Cefotaxime or ceftriaxone: & 14 \\
\hline & Tazocillin: & 7 \\
\hline & Amoxicillin: & 8 \\
\hline & Ceftazidime: & 3 \\
\hline & Oxacillin: & 2 \\
\hline & Imipenem: & I \\
\hline & Macrolides or pristinamycin: & 37 (20\%) \\
\hline & Spiramycin: & 35 \\
\hline & Erythromycin: & I \\
\hline & Pristinamycin: & I \\
\hline & Fluoroquinolones: & 20 (1 I\%) \\
\hline & Ofloxacin: & 14 \\
\hline & Ciprofloxacin: & 6 \\
\hline & Cotrimoxazole: & $8(4 \%)$ \\
\hline & Aminoglycosides: & $6(3.5 \%)$ \\
\hline & Amikacin: & 5 \\
\hline & Gentamicin: & I \\
\hline & Nitro-imidazoles: & $3(2 \%)$ \\
\hline & Glycopeptides: & I (0.5\%) \\
\hline & Other classes: & $3(2 \%)$ \\
\hline \multirow[t]{7}{*}{ Infection treated } & Pneumonia: & $58(52 \%)$ \\
\hline & Bronchitis: & $21(19 \%)$ \\
\hline & Intra-abdominal: & $6(5.5 \%)$ \\
\hline & Urinary tract: & $6(5.5 \%)$ \\
\hline & Neuro-meningeal: & $6(5.5 \%)$ \\
\hline & Various: & 7 (6.25\%) \\
\hline & Unknown origin: & 7 (6.25\%) \\
\hline
\end{tabular}

Multiple logistic regression analysis showed that inappropriate treatment, fluoroquinolone usage, and a higher SAPS II score were independently associated with in-hospital mortality from the initial infection (Table 4).

\section{Discussion}

It is now widely accepted that early and appropriate antimicrobial therapy of infections has an impact on in hospital mortality in the ICU setting. To our knowledge there is no published study whose specific aim was to evaluate antimicrobial therapy prescribed on admission to the ICU and to determine the impact of its appropriateness on hospital mortality. The link between treatment inappropriateness and mortality has been clearly established for certain types of infections: ventilator-associated pneumonia, bloodstream infections, or infections complicated by severe 
Table 3 Univariate analysis of risk factors for inappropriate empirical antimicrobial therapy on admission (appreciated on 85 empirical prescriptions for correctly diagnosed infections; empirical prescriptions for misdiagnosed or undiagnosed infections $(n=9)$ and microbiologically documented prescriptions $(n=17)$ were excluded $)$

\begin{tabular}{|c|c|c|c|}
\hline Factor & $\begin{array}{l}\text { Inappropriate therapy } \\
(\mathrm{n}=9) \text { No. (\%) }\end{array}$ & $\begin{array}{l}\text { Appropriate therapy } \\
(n=76) \text { No. }(\%)\end{array}$ & p value \\
\hline SAPS II score & $45.8 \pm 18.2$ & $39.0 \pm 18.7$ & 0.22 \\
\hline Sex (male) & $8(89)$ & $45(59)$ & 0.14 \\
\hline Age & $56 \pm 12$ & $58 \pm 18$ & 0.99 \\
\hline Mechanical ventilation & $6(67)$ & $38(50)$ & 0.49 \\
\hline Noninvasive ventilation & $2(22)$ & $10(13)$ & 0.61 \\
\hline Dopamine use & $0(0)$ & $3(4)$ & 1.00 \\
\hline Epinephrine or norepinephrine use & $3(33)$ & $9(12)$ & 0.11 \\
\hline Hospital-acquired infection & $4(44)$ & II (14) & $0.0475^{*}$ \\
\hline Delay between admission and treatment (in hours) & $3.67 \pm 2.45$ & $4.30 \pm 3.97$ & 0.98 \\
\hline Treatment during the night & $4(44)$ & $25(33)$ & 0.48 \\
\hline Ongoing antimicrobial treatment on admission: No & $6(67)$ & $51(67)$ & 0.74 \\
\hline Yes and unchanged & $0(0)$ & $7(9)$ & \\
\hline Yes and modified & $3(33)$ & $18(24)$ & \\
\hline Use of tazocillin, ceftazidim or imipenem & $2(22)$ & $4(5)$ & 0.12 \\
\hline Other betalactam usage & $7(78)$ & $67(88)$ & 0.33 \\
\hline Aminoglycoside usage & $0(0)$ & $2(3)$ & 1.00 \\
\hline Fluoroquinolone usage & $2(22)$ & $7(9)$ & 0.24 \\
\hline Macrolide or pristinamycin usage & $6(67)$ & $29(38)$ & 0.15 \\
\hline Use of other antibiotics & $2(22)$ & $8(11)$ & 0.29 \\
\hline Prior hospitalization in the preceding year & $6(67)$ & $32(43)$ & 0.29 \\
\hline Patients receiving antibiotics before admission & $2(22)$ & $34(45)$ & 0.29 \\
\hline Pneumonia or pleural fluid infection & $7(78)$ & $44(58)$ & 0.30 \\
\hline Urinary tract infection & $\mathrm{I}(\mathrm{II})$ & $\mathrm{I}(\mathrm{I})$ & 0.20 \\
\hline Other infection & I (II) & $3 I(4 I)$ & 0.14 \\
\hline
\end{tabular}

sepsis or septic shock. ${ }^{1-12}$ In the case of ventilator-associated pneumonia, it has even been shown that modifying an initial inappropriate therapy according to the microbiological documentation obtained by bronchoalveolar lavage does not translate in a better outcome. ${ }^{3}$ Thus it appears that the efficacy of initial therapy, and notably of initial therapy on admission to the ICU is crucial for clinical outcome and is more important than adaptation of the treatment to microbiological results.

In the present work, we first assessed the different aspects of antibiotic prescription in the 24 hours following admission in our ICU. We found that $34 \%$ of the patients received antimicrobial treatment within this time period. But in fact $29 \%$ of the patients admitted were really infected at admission which is nonetheless almost one third of the patients. This result is similar to that reported (21\%) in the international multicenter study from Alberti and colleagues. ${ }^{12}$
This confirms that infection at admission to the ICU in an important issue. Another important point of our study is that $85 \%$ of the treatments were empirically prescribed, only $15 \%$ of the infections being microbiologically documented on admission. Similar results (14\%) have been obtained in another study performed in one ICU. ${ }^{13}$ This stresses the importance of the appropriateness of initial empirical antibiotic therapy in the ICU setting.

To achieve that goal, one needs to be helped by local empirical treatment guidelines adapted to the susceptibility of the microorganisms and regularly reevaluated. In ventilatorassociated pneumonia, it has been shown that the application of an empirical treatment guideline can increase the initial administration of appropriate antimicrobial treatment. ${ }^{14}$ In our hospital, local empirical prescription guidelines are available for almost all types of infections and we thus wanted to determine whether they were followed; if they were not, for 
Table 4 A Univariate analysis of risk factors for in-hospital mortality due to the initial infection (I07 patients). B) Risk factors independently associated with in-hospital mortality due to to the initial infection

\begin{tabular}{|c|c|c|c|}
\hline \multirow[t]{2}{*}{ Factor } & \multicolumn{3}{|c|}{ In-hospital mortality from the initial infection } \\
\hline & Yes $(n=13)$ & No. $(n=94)$ & p value \\
\hline SAPS II score (mean \pm SD) & $58.7 \pm 25.5$ & $38.9 \pm 18.0$ & $0.005^{*}$ \\
\hline Sex (male) & $10(77)$ & $57(6 \mathrm{I})$ & 0.36 \\
\hline Age & $66 \pm 14$ & $59 \pm 17$ & 0.14 \\
\hline Mechanical ventilation & $8(62)$ & $46(49)$ & 0.56 \\
\hline Noninvasive ventilation & $2(15)$ & $8(9)$ & 0.35 \\
\hline Dopamine use & I (8) & $5(5)$ & 0.55 \\
\hline Epinephrine or norepinephrine use & $8(62)$ & $10(11)$ & $<0.00$ I $^{*}$ \\
\hline Hospital-acquired infection & $6(46)$ & $19(20)$ & 0.07 \\
\hline $\begin{array}{l}\text { Delay (hours) between admission and treatment } \\
\text { (mean } \pm \text { SD) }\end{array}$ & $4.5 \pm 2.6$ & $4.5 \pm 4.2$ & 0.40 \\
\hline Treatment during the night & $6(46)$ & $32(30)$ & 0.35 \\
\hline Ongoing antimicrobial treatment on admission: No & $5(38)$ & $61(65)$ & 0.13 \\
\hline Yes and unchanged & $2(15)$ & $8(9)$ & \\
\hline Yes and modified & $6(46)$ & $25(27)$ & \\
\hline Use of tazocillin, ceftazidim or imipenem & $4(3 I)$ & $6(6)$ & $0.02 *$ \\
\hline Use of other beta-lactams & $10(77)$ & $80(85)$ & 0.43 \\
\hline Use of aminoglycosides & $2(15)$ & $4(4)$ & 0.15 \\
\hline Use of fluoroquinolones & $7(54)$ & $12(13)$ & $0.002^{*}$ \\
\hline Use of macrolides or pristinamycin & $3(23)$ & $35(37)$ & 0.37 \\
\hline Use of other antibiotics & $0(0)$ & $14(15)$ & 0.21 \\
\hline Prior hospitalization in the preceding year & $8(62)$ & $43(46)$ & 0.38 \\
\hline Pneumonia of pleural fluid infection & $5(38)$ & $55(59)$ & 0.16 \\
\hline Urinary tract infection & $2(15)$ & $4(4)$ & 0.15 \\
\hline Other infection & $4(31)$ & $34(36)$ & 0.77 \\
\hline Bacteremia & $3(23)$ & $8(9)$ & 0.13 \\
\hline Microbiological documentation at 48 hours & $7(54)$ & $40(43)$ & 0.42 \\
\hline Origin of the patient: Emergency unit & $3(23)$ & $45(48)$ & 0.17 \\
\hline Other unit of the hospital & $8(62)$ & $33(35)$ & \\
\hline Community & $2(15)$ & $16(17)$ & \\
\hline Appropriate or excessive empirical treatment & $4(31)$ & $69(73)$ & $0.002 *$ \\
\hline Inappropriate empirical treatment & $4(31)$ & $5(5)$ & \\
\hline $\begin{array}{l}\text { Empirical treatment without localization } \\
\text { of the infection source }\end{array}$ & $3(23)$ & $6(6)$ & \\
\hline Microbiologically documented treatment & $2(15)$ & $14(15)$ & \\
\hline \multicolumn{4}{|l|}{ B) } \\
\hline Risk factor & OR (95\% Cl) & p value & \\
\hline Inappropriate treatment & 14.64 (2.17-98.97) & 0.006 & \\
\hline Use of fluoroquinolones & $8.22(1.88-35.95)$ & 0.005 & \\
\hline Increasing SAPS II score (one-point increments) & $1.04(1.01-1.07)$ & 0.02 & \\
\hline
\end{tabular}

Abbreviations: $\mathrm{Cl}$, confidence interval; OR, odds ratio; SAPS II, simplified acute physiology score; SD, standard deviation.

which reasons; and if nonadherence to them had an impact on in-hospital mortality due to the initial infection.

Compared to the works that have already been performed and which were based on microbiological documentation, an advantage of our definition of treatment appropriateness is that it permits to evaluate the treatments of infections which remain undocumented, and this represented 50\% of the cases in our study. This relatively high percentage could be explained by one main reason: $76 \%$ of the infections we investigated were community-acquired and Alberti 
and colleagues clearly showed that the documentation rates are lower for community-acquired infections compared to hospital-acquired or ICU-acquired infections. ${ }^{12}$ In their international prospective cohort study of 3034 infectious episodes, the documentation rates were $86 \%, 71 \%$, and $55 \%$ for ICU-acquired, hospital-acquired, and community-acquired infections, respectively. Furthermore, the nonevaluation of undocumented infections could represent a bias, because infections due to microorganisms difficult to identify are therefore for the most part not taken into account in the evaluation. On the other hand, with our definition of treatment appropriateness infections microbiologically documented on admission could not be evaluated. But they represented only a small percentage of the cases included (15\%). Furthermore, it is clear that the risk of inappropriate treatment is lower when microbiological documentation is available.

We found that $89 \%$ of the initial empirical prescriptions were appropriate. The percentages of appropriateness found by other workers are similar to ours (from $83 \%$ to $99 \%$ ) in some studies ${ }^{8,11,13}$ and lower (70\% to $77 \%$ ) in others. ${ }^{6,9,15}$ However, our results are difficult to compare with those of other works because, in all these studies appropriateness was judged according to microbiological documentation.

We then wanted to assess which factors were associated with treatment inappropriateness and whether our definition of treatment inappropriateness based on treatment guidelines had an impact on in-hospital mortality due to the initial infection. By univariate analysis, the only factor associated with empirical inappropriate prescriptions was the hospitalacquired characteristics of the infection treated. Examination of the cases showed that the main cause for this association was that the hospital-acquired character of the infection was not taken into account in the initial prescription, and antibiotic-resistant hospital-acquired organisms were therefore not covered by the prescription.

Concerning the link with mortality due to the initial infection, we found by multivariate analysis that the risk of in-hospital mortality from the initial infection was more than 14 times higher in case of inappropriate empirical treatment. However, our work suffers from some limitations: first, it is a monocentric study; second the number of cases evaluated was relatively low leading to large confidence intervals; third, the way appropriateness was judged depended on our local prescription guidelines and cannot be generalized to other hospitals.

As regards to the types of infections included, the study performed by Kollef and colleagues ${ }^{15}$ is the only one that can be compared with our work. They performed a prospective cohort monocentric study to evaluate the relationship between inappropriate antimicrobial treatment of infections (all infections included) and hospital mortality. By multivariate analysis they also found that inappropriate antimicrobial treatment was the most important independent determinant of hospital mortality (adjusted OR 4.27, 95\% CI: 3.35-5.44; $\mathrm{p}<0.001)$. However, two major differences exist between the two studies: First, Kollef and colleagues did not restrict their study to patients already infected on admission but also included ICU-acquired infections. Second, their definition of treatment inappropriateness was the classical one used in all studies so far performed: prescription of at least one antibiotic active against the microorganism(s) isolated.

The other independent risk factor for hospital mortality that we identified was prescription of fluoroquinolones. Nseir and colleagues showed that fluoroquinolone use was an independent risk factor for subsequent acquisition of multidrug resistant bacteria in the ICU by the patient. ${ }^{16}$ We did not observe this phenomenon among the seven patients who received fluoroquinolones and who died. But we observed that four of them were treated for hospital-acquired infections and in three of them treatment was inappropriate because the hospital-acquired character of the infection was not taken into account. Thus, fluoroquinolone-resistant organisms could have been implicated; but unfortunately these cases were not microbiologically documented. Concerning the three remaining patients treated with fluoroquinolones for communityacquired infections and then died, we found a link between the prescription and death for one patient: he presented with acute prostatitis and was treated empirically with cefotaxime and ofloxacin. This infection was secondarily microbiologically documented with a strain of Enterocococcus faecalis being isolated from blood and urine. It is well known that this species is resistant to both third-generation cephalosporins and fluoroquinolones. Fluoroquinolone prescription was not implicated in the death of the two other patients. They presented with bacteremia due to fluoroquinolone-susceptible Staphylococcus aureus and their death was attributable to the severity of the sepsis in both cases.

Our work also showed that infections of undetermined origin are not rare and carry a high mortality rate. This had already been observed in other studies which dealt with ICU patients suffering from severe infections: sepsis or septic shock. ${ }^{8,17}$ One likely explanation is that, when treating an occult infectious process, most physicians are reluctant to prescribe potent broad-spectrum agents immediately. This emphasizes the need for an aggressive search for a focus of infection in all infected patients admitted to the ICU. 
This study is thus the first to show that nonadherence to local empirical antibiotic prescription guidelines for patients infected on ICU admission is independently associated with increased in-hospital mortality due to the initial infection. More studies are needed to confirm our findings.

\section{Disclosure}

The authors report no conflicts of interest in this work.

\section{References}

1. Iregui M, Ward S, Sherman G, et al. Clinical importance of delays in the inititiation of appropriate antibiotic treatment for ventilator-associated pneumonia. Chest. 2002;122:262-268.

2. Kollef MH, Ward S. The influence of mini-BAL cultures on patient outcomes: implications for the antibiotic management of ventilatorassociated pneumonia. Chest. 1998;113:412-420.

3. Luna CM, Vujacich P, Niedermann MS, et al. Impact of BAL data on the therapy and outcome of ventilator-associated pneumonia. Chest. 1997;111:676-685.

4. Rello J, Gallego M, Mariscal D, et al. The value of routine microbial investigation in ventilator-associated pneumonia. Am J Respir Crit Care Med. 1997;156:196-200.

5. Bryan CS, Reynolds KL, Brenner ER. Analysis of 1186 episodes of Gram-negative bacteremia in non-university hospitals: the effects of antimicrobial therapy. Rev Infect Dis. 1983;5:629-638.

6. Ibrahim EH, Sherman G, Ward S, et al. The influence of inadequate antimicrobial treatment of bloodstream infections on patient outcomes in the ICU setting. Chest. 2000;118:146-155.

7. Lodise TP, McKinnon PS, Swiderski L, et al. Outcomes analysis of delayed antibiotic treatment for hospital-acquired Staphylococcus aureus bacteremia. Clin Infect Dis. 2003;36:1418-1423.
8. Garnacho-Montero J, Garcia-Garmendia JL, Barrero-Almodovar A, et al. Impact of adequate empirical antibiotic therapy on the outcome of patients admitted to the intensive care unit with sepsis. Crit Care Med. 2003;31:2742-2751.

9. Harbarth S, Garbino J, Pugin J, et al. Inappropriate initial antimicrobial therapy and its effect on survival in a clinical trial of immunomodulating therapy for severe sepsis. Am J Med. 2003;115:529-535.

10. Kumar A, Roberts D, Wood KE, et al. Duration of hypotension before initiation of effective antimicrobial therapy is the critical determinant of survival in human septic shock. Crit Care Med. 2006;34:1589-1596.

11. Leone M, Bourgoin A, Cambon S, et al. Empirical antimicrobial therapy of septic shock patients: adequacy and impact on the outcome. Crit Care Med. 2003;21:462-467.

12. Alberti C, Brun-Buisson C, Burchardi H, et al. Epidemiology of sepsis and infection in ICU patients from an international multicentre cohort study. Intensive Care Med. 2002;28:108-121.

13. Montay V, Alfandari S, Senneville B, et al. Evaluation de la prescription antibiotique dans un service de réanimation polyvalente. Presse Médicale. 1998;27:700-704.

14. Ibrahim EH, Ward S, Sherman G, et al. Experience with a clinical guideline for the treatment of ventilator-associated pneumonia. Crit Care Med. 2001;29:1109-1115.

15. Kollef MH, Sherman G, Ward S, et al. Inadequate antimicrobial treatment of infections: a risk factor for hospital mortality among critically ill patients. Chest. 1999;115:462-474.

16. Nseir S, Di Pompeo C, Soubrier S, et al. First-generation fluoroquinolone use and subsequent emergence of multidrug-resistant bacteria in the intensive care unit. Crit Care Med. 2005;33:289-289.

17. Reyes WJ, Brimioulle S, Vincent JL. Septic shock without documented infection: an uncommon entity with a high mortality. Intensive Care Med. 1999;25:1267-1270.
Therapeutics and Clinical Risk Management

\section{Publish your work in this journal}

Therapeutics and Clinical Risk Management is an international, peerreviewed journal of clinical therapeutics and risk management, focusing on concise rapid reporting of clinical studies in all therapeutic areas, outcomes, safety, and programs for the effective, safe, and sustained use of medicines. This journal is indexed on PubMed Central, CAS,

\section{Dovepress}

EMBase, Scopus and the Elsevier Bibliographic databases. The manuscript management system is completely online and includes a very quick and fair peer-review system, which is all easy to use. Visit http://www.dovepress.com/testimonials.php to read real quotes from published authors. 